\title{
Luto em cuidadores: realidade de dois Agrupamentos de Centros de Saúde do Norte
}

Soraia Santos, ${ }^{1-2}$ Carla Lopes da Mota, ${ }^{3-4}$ Isabel Chaves e Castro, ${ }^{5}$ Paula Sapeta ${ }^{6}$

\section{RESUMO}

Objetivo: Caracterizar os cuidadores nos doze meses após a morte do doente (dados sociodemográficos, acompanhamento clínico, problemas de saúde e terapêutica farmacológica).

Métodos: Realizou-se um estudo observacional, retrospetivo e analítico, ao nível dos cuidados de saúde primários. A população era constituída por cuidadores de doentes falecidos no ano de 2017 , identificados pelo médico de família dos últimos. Após envio de convite à participação de todas as Unidades Funcionais foram obtidos os dados que permitiram caracterizar os cuidadores e o acompanhamento clínico no primeiro ano de luto, através dos médicos de família que aceitaram participar. Para a análise descritiva foram utilizadas frequências absolutas e relativas, mediana, mínimo e máximo.

Resultados: Dos 308 cuidadores destaca-se a representatividade dos idosos (mediana de idade - 66,5 anos) e das mulheres (79,2\%), bem como o acompanhamento exclusivo pelo médico de família em $93,2 \%$ das situações. A prescrição de novo e/ou alteração de dosagem de psicotrópicos ocorreu em 35,1\% dos cuidadores, contrastando com a baixa taxa de acompanhamento em psicologia $(3,5 \%)$ e/ou psiquiatria $(4,2 \%)$ e ausência de avaliação de risco no luto e fornecimento de ferramentas de apoio.

Conclusão: As características dos cuidadores foram concordantes com as descritas na literatura. A medicalização do luto pode refletir escassez de formação, tempo de consulta e de recursos de psicologia e psiquiatria. Comprova-se o papel essencial do médico de família no apoio ao luto; a implementação de medidas institucionais e organizacionais pode melhorar a prestação de cuidados no luto.

Palavras-chave: Cuidados de saúde primários; Luto; Cuidadores; Médicos de família.

\section{INTRODUÇÃO}

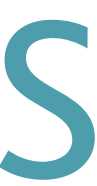
egundo o modelo cooperativo com intervenção nas crises, os cuidados paliativos implicam, para além dos cuidados ao doente, o apoio e o cuidar da sua família durante o fim de vida, prolongando-se após a morte, nomeadamente no luto. ${ }^{1}$ Este poderá ser definido como uma "reação característica a uma perda significativa”, em que a perda pode ser, por exemplo, de uma pessoa ou de uma expectativa. ${ }^{1}$ Esta perda poderá ter características comuns, mas terá sempre uma componente individual, uma vez que cada pessoa é única - "eu sou eu e a minha circunstância", citação de Ortega e Gasset por Lucília Nunes. ${ }^{2}$

O cuidador informal pode ser definido como um familiar, amigo ou vizinho que presta apoio diário, não remunerado, a uma pessoa incapaz de realizar as atividades inerentes à vida diária. ${ }^{3}$ Este papel pode gerar im- pacto emocional, físico e económico, que poderá levar a uma menor qualidade de vida, ${ }^{3-4}$ ao aumento de prevalência de depressão e sintomas do foro emocional ${ }^{5-6} \mathrm{e}$ também ao desenvolvimento de complicações no luto. ${ }^{7}$

Em Portugal foi publicado o Estatuto do Cuidador Informal, em setembro de 2019, reconhecendo o

1. Médica Assistente de Medicina Geral e Familiar. ACeS Grande Porto VIII - Espinho/Gaia, Vila Nova de Gaia.

2. Membro do Grupo de Estudos de Cuidados Paliativos. Associação Portuguesa de Medicina Geral e Familiar.

3. Médica Assistente de Medicina Geral e Familiar. Coordenadora da Equipa Comunitária de Suporte em Cuidados Paliativos. ACeS Grande Porto VII - Gaia, Vila Nova de Gaia.

4. Membro da Comissão Coordenadora do Grupo de Estudos de Cuidados Paliativos. Associação Portuguesa de Medicina Geral e Familiar.

5. Médica Assistente Graduada Sénior de Medicina Geral e Familiar, na Equipa Comunitária de Suporte em Cuidados Paliativos. ACeS Grande Porto VII - Gaia, Vila Nova de Gaia.

6. Professora e Coordenadora do Mestrado em Cuidados Paliativos. Escola Superior Dr. Lopes Dias, Instituto Politécnico de Castelo Branco. 
impacto que a prestação de cuidados tem no cuidador e, desta forma, regulando os direitos e os deveres do cuidador e da pessoa cuidada, estabelecendo as respetivas medidas de apoio. ${ }^{8}$

A maioria dos cuidadores terá um luto «normal», adaptativo, conquanto alguns terão uma resposta não adaptativa, prolongada, que poderá exacerbar problemas físicos, psicológicos e sociais. ${ }^{9-12}$ Estima-se que o luto complicado/prolongado/patológico afete 10-20\% dos enlutados, ${ }^{10,13-15}$ embora um estudo português, de 2015, tenha identificado, em cuidadores de doentes acompanhados em cuidados paliativos, uma prevalência de luto complicado de $28,8 \% .^{3}$ Este luto complicado/prolongado/patológico é designado na DSM-V como Perturbação do Luto Complexo Persistente. ${ }^{16}$ Esta distingue-se do luto normal pela presença de reações graves de luto que se mantêm por, pelo menos, doze meses (ou seis meses em crianças) após a morte da pessoa próxima. O transtorno é diagnosticado somente quando persistem níveis graves de resposta de luto pelo período indicado, interferindo na capacidade do indivíduo de funcionar, sendo fatores de risco para o seu desenvolvimento ser do sexo feminino, idoso, com antecedentes psiquiátricos, morte do filho ou cônjuge ou morte do súbita do ente querido, entre outros. ${ }^{17}$

A evidência parece demonstrar que o apoio no luto deverá ser oferecido a todos os enlutados, sendo que o tipo de apoio deverá ser diferenciado de acordo com as necessidades evidenciadas, nomeadamente perante a presença de fatores de risco para luto complicado ou de sinais de patologia psiquiátrica, tendo sempre presente que os indivíduos de baixo risco poderão não beneficiar de ações mais complexas. ${ }^{12-14}$ Assim, a maioria dos enlutados apresentará necessidades de nível baixo a intermédio, estando indicado o apoio não especializado, ${ }^{18}$ pelo que outros profissionais de saúde, como os médicos de família e os enfermeiros, serão fundamentais para o assegurar. ${ }^{12} \mathrm{O}$ documento conjunto da European Association for Palliative Care e daWONCA sustenta o apoio aos cuidadores/familiares e prestação de apoio no luto como uma das áreas de intervenção dos médicos de família. ${ }^{19}$

Em Portugal é desconhecido qual o apoio prestado pelos médicos de família aos cuidadores em luto, bem como o modo como estes utilizam os cuidados de saúde, a incidência de problemas de saúde e o recurso à terapêutica farmacológica após a morte do doente.
Com este estudo pretende-se, assim, caracterizar os cuidadores nos doze meses após a morte do doente de quem cuidavam, no que se refere a características sociodemográficas, acompanhamento clínico no luto, problemas de saúde e terapêutica farmacológica.

\section{MÉTODOS}

Realizou-se um estudo observacional, retrospetivo e analítico, entre novembro de 2018 e agosto de 2019, em dois Agrupamentos de Centros de Saúde (ACeS) da Administração Regional de Saúde (ARS) do Norte: Grande Porto VII - Gaia e Grande Porto VIII - Espinho/Gaia.

A população em estudo era constituída pelos cuidadores dos doentes falecidos no ano de 2017, identificados como tal pelo médico de família dos últimos. Foram incluídos cuidadores com, pelo menos, 18 anos de idade, que se encontrassem inscritos, tal como o doente, num dos ACeS, na mesma unidade e com médico de família atribuído. Definiram-se como critérios de exclusão: cuidadores de doentes falecidos que não tenham tido contacto (presencial e/ou não presencial) com o médico de família nos doze meses que antecederam a sua morte ou cujo médico de família tenha sido atribuído por um período inferior a doze meses prévios à morte; cuidadores sem contacto (presencial e/ou não presencial) com médico de família nos três anos prévios à morte do doente ou sem consulta médica presencial nos doze meses após; cuidador principal desconhecido; médico de família indisponível.

Realizou-se um censo, com contagem exaustiva, identificando todos os óbitos ocorridos em 2017 em cada um dos ACeS envolvidos. ${ }^{20}$ Após este processo, contactaram-se, via email, todos os coordenadores das Unidades Funcionais - Unidade de Saúde Familiar (USF) e Unidade de Cuidados de Saúde Personalizados (UCSP) -, endereçando um convite à participação de todos os médicos de família. Aos que aceitaram, após identificação dos óbitos na lista de utentes que lhes estava atribuída, solicitou-se a identificação do cuidador principal do doente falecido e o cumprimento dos pressupostos apresentados no fluxograma (Figura 1).

Nos casos em que se aplicavam os critérios de inclusão e nenhum dos critérios de exclusão obtiveramse os seguintes dados: caracterização do cuidador; caracterização do doente falecido; acompanhamento clínico, problemas de saúde identificados e terapêutica 


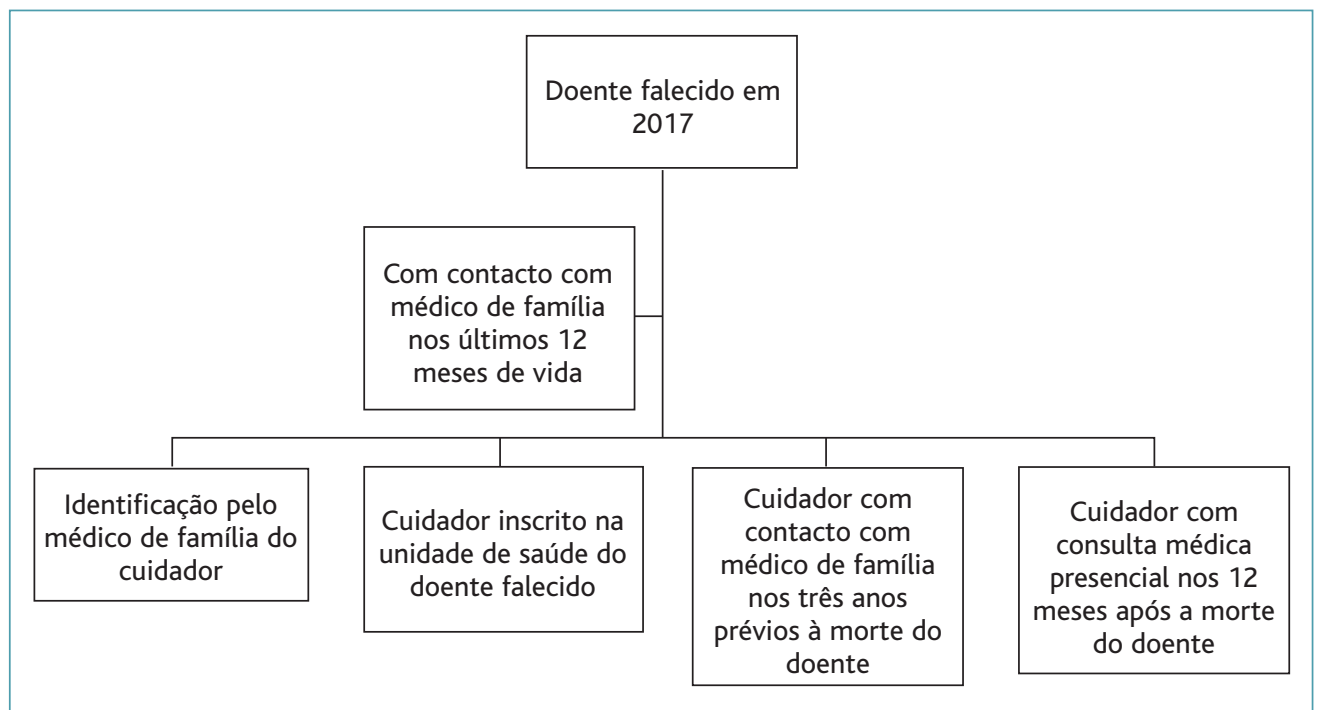

Figura 1. Fluxograma de seleção de participantes.

farmacológica, de novo ou com dosagem alterada, do cuidador nos doze meses após a morte do doente.

Os problemas de saúde foram definidos de acordo com a codificação da Classificação Internacional dos Cuidados de Saúde Primários - 2a edição (ICPC-2). ${ }^{21}$

Construiu-se uma base de dados e realizou-se o tratamento dos dados através do programa informático Statistical Package for the Social Sciences ${ }^{\circledR}$, v. 26.

Avaliou-se a normalidade da distribuição da amostra nas variáveis quantitativas através do teste Kolmogorov-Smirnov.

Procedeu-se a uma análise descritiva, utilizando para as variáveis qualitativas as frequências absolutas e relativas e para as variáveis quantitativas a mediana, mínimo e máximo, quando a distribuição não cumpria os princípios da normalidade. Na análise estatística inferencial foram utilizados os testes de hipóteses $\chi^{2}$ para as variáveis qualitativas e Mann-Whitney e Kruskal-Wallis para as variáveis quantitativas sem distribuição normal.

Definiu-se um nível de significância de 0,05. As variáveis que demonstraram um nível de significância menor ou igual a 0,08 foram analisadas num modelo de regressão logística.

As regras de conduta ética e de boas práticas foram sempre acauteladas para que fossem cumpridos os preceitos da Declaração de Helsínquia. ${ }^{22}$

Obtiveram-se pareceres favoráveis dos Conselhos Clínicos e de Saúde do ACeS Gaia e do ACeS Espi-
nho/Gaia, formalizados pelos seus Presidentes do Conselho Clínico e da Saúde, e da Comissão de Ética para a Saúde da ARS do Norte.

\section{RESULTADOS}

Em 2017, os ACeS Gaia e Espinho/Gaia eram constituídos por 12 e 23 unidades de saúde (respetivamente, sete - USF modelo A, 13 - USF modelo B, 15 - UCSP), que abrangiam uma população residente de 329.561 habitantes, representando cerca de 9,2\% da população da região Norte, em $2016 .^{23}$

Faleceram 2.653 indivíduos inscritos nestes ACeS no ano de 2017; 19,3\% dos óbitos ocorreram em doentes inscritos nas USF modelo A, 53,0\% em USF modelo B e 27,7\% em UCSP.

Aceitaram participar 61 médicos de família, que tinham ao seu cuidado 844 doentes falecidos em 2017 (31,8\% do total de óbitos ocorridos nos ACeS estudados).

Considerando os utentes dos médicos de família que participaram no estudo, cerca de $80,5 \%(n=679)$ dos doentes que faleceram tiveram algum tipo de contacto com o seu médico de família nos 12 meses anteriores à sua morte (87,5\% nas UCSP; $75,4 \%$ nas USF modelo A; 81,4\% nas USF modelo B). Não existiu diferença estatisticamente significativa entre as diferentes tipologias de unidade funcional [USF modelo B $v s$ UCSP ( $p=0,336)$; USF modelo A $v s$ UCSP $(p=0,100)$; USF modelo A vs USF Modelo B $(p=0,075)]$.

Os médicos de família participantes identificaram 349 cuidadores, o que corresponde a $51,4 \%$ dos doentes com contacto com o médico de família nos últimos 12 meses de vida.

Quarenta e um cuidadores não tiveram contacto com o seu médico de família nos três anos prévios à morte ou consulta presencial nos 12 meses após, pelo que foram excluídos, tendo-se obtido 308 indivíduos para inclusão no estudo. Este valor corresponde a cui- 
TABELA 1. Caracterização dos cuidadores

\begin{tabular}{|c|c|c|c|}
\hline \multicolumn{2}{|l|}{$\mathrm{ACeS}, n(\%)$} & \multicolumn{2}{|l|}{ Tipo de família, $\boldsymbol{n}(\%)$} \\
\hline Gaia & $114(37,0 \%)$ & Nuclear & $59(19,2 \%)$ \\
\hline Espinho/Gaia & $194(63,0 \%)$ & Alargada & $22(7,1 \%)$ \\
\hline Unidade Funcional, n (\%) & & Unitária & $120(39,0 \%)$ \\
\hline UCSP & $18(5,8 \%)$ & Monoparental & $25(8,1 \%)$ \\
\hline USF A & $52(16,9 \%)$ & Outra & $16(5,2 \%)$ \\
\hline \multirow[t]{2}{*}{ USF B } & $238(77,3 \%)$ & Desconhecido & $64(20,8 \%)$ \\
\hline & & Parentesco, $n(\%)$ & \\
\hline \multirow[t]{3}{*}{ Idade cuidador, Md (Mín; Máx) } & $66,5(25 ; 91)$ & Cônjuge & $174(56,5 \%)$ \\
\hline & & Filho/a & $104(33,8 \%)$ \\
\hline & & Pai/Mãe & $8(2,6 \%)$ \\
\hline Sexo, $n(\%)$ & & Irmão/ã & $7(2,3 \%)$ \\
\hline Masculino & $64(20,8 \%)$ & Sobrinho/a & $3(1,0 \%)$ \\
\hline \multirow[t]{2}{*}{ Feminino } & $244(79,2 \%)$ & Outro & $11(3,6 \%)$ \\
\hline & & Desconhecido & $1(0,3 \%)$ \\
\hline Estado civil, $n$ (\%) & & Cuidador único, $n(\%)$ & \\
\hline Solteiro/a & $28(9,1 \%)$ & Não & $183(59,4 \%)$ \\
\hline Casado/a ou união de facto & $62(20,1 \%)$ & Sim & $76(24,7 \%)$ \\
\hline Viúvo/a & $180(58,4 \%)$ & Desconhecido & $49(15,9 \%)$ \\
\hline \multirow{2}{*}{$\begin{array}{l}\text { Divorciado/a } \\
\text { Desconhecido }\end{array}$} & $8(2,6 \%)$ & & \\
\hline & $30(9,7 \%)$ & $N^{\circ}$ fatores de risco para PLP, Md (Mín, Máx) & $3,0(0 ; 10)$ \\
\hline \multicolumn{2}{|l|}{ Habilitações literárias, $\boldsymbol{n}(\%)$} & \multicolumn{2}{|l|}{ Tempo de prestação de cuidados } \\
\hline$<4$ anos & $49(15,9 \%)$ & em meses, Md (Mín, Máx) & $30,3(0 ; 395)$ \\
\hline \multirow{2}{*}{$\begin{array}{l}4 \text { a } 12 \text { anos } \\
>12 \text { anos }\end{array}$} & $124(40,3 \%)$ & & \\
\hline & $21(6,8 \%)$ & \multicolumn{2}{|l|}{ Local de prestação cuidados, $n$ (\%) } \\
\hline Desconhecido & $114(37,0 \%)$ & Residência de ambos & $221(71,8 \%)$ \\
\hline Situação profissional, $n$ (\%) & & Residência do cuidador & $\begin{array}{r}32(10,4 \%) \\
28(9,1 \%)\end{array}$ \\
\hline Inativo & $191(62,0 \%)$ & Residência de um familiar & $1(0,3 \%)$ \\
\hline Ativo & $115(37,3 \%)$ & Outra & $2(0,6 \%)$ \\
\hline Desconhecido & $2(0,6 \%)$ & Desconhecido & $24(7,8 \%)$ \\
\hline
\end{tabular}

Legenda: $n$ = frequência absoluta; \% = frequência relativa; Md = mediana; Mín = mínimo; Máx = máximo; PLP = Perturbação de Luto Prolongado.

dadores de 11,6\% dos óbitos ocorridos nos dois ACeS.

Nenhuma das variáveis contínuas apresentou distribuição normal.

A maioria dos cuidadores estava inscrito no ACeS Espinho/Gaia (63,0\%) e em unidades de saúde com tipologia USF modelo B $(77,3 \%)$ (Tabela 1$)$.

A mediana da idade dos cuidadores foi de 66,5 anos, sendo que $79,2 \%$ eram mulheres, a maioria tinha como estado civil o de viúvo (58,4\%), $40 \%$ cumpriram quatro a 12 anos de escolaridade, $62 \%$ eram profissionalmen- te inativos, $39 \%$ viviam sozinhos e $59,4 \%$ não eram cuidadores únicos. No que se refere ao parentesco, $56,5 \%$ eram cônjuges e 33,8\% filhos do doente falecido. A mediana de fatores de risco para Perturbação de Luto Prolongado (PLP) ${ }^{17}$ foi de três; a mediana do tempo de prestação de funções foi de 30 meses (43,3\% dos cuidadores prestou cuidados durante menos de um ano e $24,7 \%$ por mais de três anos) e em 71,8\% das situações o local de prestação de cuidados era a residência do cuidador e do doente (Tabela 1). 


\section{TABELA 2. Caracterização dos doentes falecidos}

\section{Sexo do doente, $n$ (\%) \\ Masculino \\ Feminino}

\begin{tabular}{l|r}
\hline Idade doente em anos, Md (Mín, Máx) & $81,0(15 ; 100)$ \\
\hline Causa de morte, $\mathbf{n}(\%)$ & \\
Doença oncológica & $98(31,8 \%)$ \\
Insuficiência de órgão & $94(30,5 \%)$ \\
Doença neurodegenerativa & $56(18,2 \%)$ \\
Outra & $9(2,9 \%)$ \\
Morte súbita & $4(1,3 \%)$ \\
Desconhecido & $47(15,3 \%)$
\end{tabular}

Legenda: $n=$ frequência absoluta; \% = frequência relativa; $M d=$ mediana; Mín = mínimo; Máx = máximo.

Dos doentes falecidos 57,5\% eram homens, sendo que a mediana da idade foi de 81,0 anos. Em $31,8 \%$ dos casos a causa de morte foi doença oncológica e em $30,5 \%$ insuficiência de órgãos, desconhecendo-se a causa de morte do doente em $15,3 \%$ das situações (Tabela 2).

Relativamente ao acompanhamento clínico ao cuidador nos 12 meses após a morte do doente, $93,2 \%$ dos cuidadores foram acompanhados apenas pelo seu médico de família. Treze $(4,2 \%)$ foram acompanhados em psiquiatria e 11 (3,5\%) em psicologia. Cinco cuidadores foram simultaneamente acompanhados pelo médico de família, em psiquiatria e em psicologia (1,6\%). A mediana do número de consultas presenciais na unidade de saúde, no período analisado, foi de quatro. Cerca de $95 \%$ dos cuidadores que tiveram consulta recorreram a consulta programada e $56,8 \%$ a consulta do próprio dia; $22(7,1 \%)$ utilizaram as três tipologias de cuidados: consulta programada, consulta do próprio dia e serviço de urgência (Tabela 3).

Cerca de $7 \%$ dos cuidadores estiveram internados nos 12 meses após o falecimento do doente; em 63,6\% das situações o motivo de internamento foi por causa cirúrgica.

Em nenhum cuidador existiu aplicação de instrumento de avaliação de PLP ou uso de instrumento de apoio ao luto (carta, folheto ou outro). Em $82,5 \%$ dos cuidadores não foi efetuada nenhuma referenciação e em 15,3\% ocorreu referenciação para uma especialida-
TABELA 3. Acompanhamento clínico do cuidador nos

12 meses após a morte do doente (\% do total de

cuidadores, com exceção dos parâmetros da variável Internamento)

Tipo de acompanhamento, $\boldsymbol{n}(\%)$

Só médico de família

Médico de família + ECP

$287(93,2 \%)$

Médico de família + Psiquiatria

$1(0,3 \%)$

Médico de família + Psicologia

$8(2,6 \%)$

Médico de família + Outro

$6(1,9 \%)$

Médico de família + Psiquiatria + Psicologia

$\mathrm{N}^{\circ}$ consultas na unidade funcional

de inscrição, Md (Mín, Máx)

Tipologia de cuidados, $\boldsymbol{n}(\%)$

Consulta programada

$1(0,3 \%)$

$5(1,6 \%)$

Consulta do próprio dia

$4,0(1 ; 17)$

Serviço de urgência

$293(95,1 \%)$

$175(56,8 \%)$

Internamento, $\boldsymbol{n}$ (\%)

$29(9,4 \%)$

Motivo cirúrgico, $n$ (\% dos internamentos)

Motivo médico (\% dos internamentos)

$22(7,1 \%)$

Motivo desconhecido (\% dos internamentos)

$14(63,6 \%)$

$7(31,8 \%)$

\begin{tabular}{ll}
\hline Aplicação de instrumento PLP, $n(\%)$ & $0(0 \%)$
\end{tabular}

Instrumento de apoio ao luto, $n$ (\%)

Referenciação, $\boldsymbol{n}$ (\%)

Nenhuma

Especialidade médica

Psicologia

Nutrição

Assistente social $0(0 \%)$

UCC

$254(82,5 \%)$

$47(15,3 \%)$

$7(2,3 \%)$

$2(0,6 \%)$

$0(0 \%)$

$1(0,3 \%)$

Legenda: $n$ = frequência absoluta; \% = frequência relativa; $E C P=$ equipa de cuidados paliativos; $M d$ = mediana; Mín = mínimo; Máx = máximo.

de médica; em dois $(0,6 \%)$ casos a especialidade médica para a qual se referenciou o cuidador foi a psiquiatria.

Foram identificados 335 novos problemas de saúde em $58,1 \%$ dos cuidadores; $11 \%$ dos problemas codificados pertenciam ao Capítulo P-Psicológico da ICPC-2, afetando 8,1\% dos cuidadores (Tabela 4).

No que se refere aos fármacos prescritos, em $58,4 \%$ dos cuidadores ocorreu a prescrição de um novo fármaco, com uma mediana de 1,0 fármacos por utente. Foram prescritos psicotrópicos de novo a $28,6 \%$ dos cuidadores e, pelo menos, um antidepressivo a $15,6 \%$. 
TABELA 4. Problemas de saúde e terapêutica

farmacológica do cuidador nos 12 meses após a morte

do doente ( $\%$ do total de cuidadores)

\begin{tabular}{l|r} 
Problemas de saúde, $\boldsymbol{n}$ (\%) & $179(58,1 \%)$ \\
Capítulo P-Psicológico & $25(8,1 \%)$ \\
\hline Fármacos novos, $\boldsymbol{n}(\%)$ & $180(58,4 \%)$ \\
Psicotrópicos & $88(28,6 \%)$ \\
Antidepressivos & $48(15,6 \%)$ \\
Ansiolíticos & $42(13,6 \%)$ \\
Outros psicotrópicos & $25(8,1 \%)$ \\
\hline Alteração dosagem de fármacos, $\boldsymbol{n}(\%)$ & $54(17,5 \%)$ \\
Psicotrópicos & $26(8,4 \%)$ \\
Antidepressivos & $15(4,9 \%)$ \\
Ansiolíticos & $9(2,9 \%)$ \\
Outros psicotrópicos & $2(0,7 \%)$ \\
\hline Fármaco novo ou alteração de & $204(66,2 \%)$ \\
dosagem, $\boldsymbol{n}$ (\%) & \\
Psicotrópicos & $108(35,1 \%)$ \\
Antidepressivos & $57(18,5 \%)$ \\
Ansiolíticos & $48(15,6 \%)$ \\
Outros psicotrópicos & $27(8,8 \%)$
\end{tabular}

Legenda: $n$ = frequência absoluta; \% = frequência relativa.

Em cerca de $14 \%$ dos indivíduos existia registo de prescrição de, pelo menos, um fármaco ansiolítico de novo.

Aproximadamente $18 \%$ dos cuidadores tiveram a dosagem dos fármacos habitualmente prescritos alterada; em $8,4 \%$ das situações a alteração foi relativa a um fármaco psicotrópico.

Em $66,2 \%$ dos cuidadores ocorreu a prescrição de um novo fármaco e/ou alteração de dosagem. Os fármacos psicotrópicos foram os visados em $35,1 \%$ dos cuidadores, sendo o subgrupo mais representado o dos antidepressivos (18,5\%).

Analisou-se a relação do acompanhamento clínico, dos problemas de saúde e da terapêutica farmacológica do cuidador nos doze meses após a morte do doente, com as características do cuidador e do doente, inicialmente de forma individual e, posteriormente, recorrendo a um modelo de regressão logística (Tabela 5).

Embora seja um parâmetro de acompanhamento clínico e não de caracterização do cuidador ou do doente, incluiu-se a variável número de consultas na análise-por se considerar que poderá representar um viés de confundimento - de forma a eliminar a sua po- tencial influência na relação entre as restantes variáveis.

A existência de, pelo menos, uma consulta programada nos doze meses após a morte do doente associava-se a quatro variáveis, mas após aplicação do modelo de regressão logística apenas a idade do cuidador e o número de consultas mantiveram uma relação estatisticamente significativa $(p=0,034 ; p<0,001)$. Os cuidadores mais velhos e com maior número de consultas apresentavam maior probabilidade de ter tido consulta programada.

Também relativamente à consulta do próprio dia, apenas as variáveis idade do cuidador e número de consultas apresentaram relação estatisticamente significativa ( $p=0,018 ; p<0,001)$. Utentes mais novos e com mais consultas apresentavam maior probabilidade de ter tido consulta do próprio dia.

Quando considerado o recurso ao serviço de urgência, as três variáveis (tipo de família, número de fatores de risco para PLP, número de consultas) que demonstraram relação significativa na análise individual mantiveram esse efeito após aplicação do modelo de regressão logística ( $p=0,033 ; p=0,020 ; p=0,001$ ). Os cuidadores inseridos em famílias alargadas e de tipologia «outras» tinham maior probabilidade de ter recorrido ao serviço de urgência, comparativamente com os inseridos em famílias monoparentais. Utentes com mais fatores de risco para PLP e maior número de consultas também apresentavam maior probabilidade de ter recorrido a este serviço.

Era mais provável ter maior número de consultas apresentando menor nível de escolaridade $(p=0,040)$; mais nenhuma variável estudada mostrou associação, estatisticamente significativa ou no limiar da significância, com o número de consultas.

Menor tempo de prestação de cuidados e maior número de consultas associavam-se à codificação de problemas de saúde de novo nos doze meses após a morte do doente, mantendo-se uma relação estatisticamente significativa quando ambas as variáveis foram consideradas num modelo de regressão logística $(p=0,020 ; p<0,001)$. Considerando os problemas de saúde apenas do capítulo P-Psicológico do ICPC-2 foi demonstrada associação significativa com o número de fatores de risco para PLP $(p=0,029)$, havendo maior probabilidade de haver codificação de um problema des- 
TABELA 5. Relação entre acompanhamento clínico/problemas de saúde/terapêutica farmacológica do cuidador nos 12 meses após a morte do doente e as características de ambos

\begin{tabular}{|c|c|c|}
\hline & Análise individual & Regressão logística \\
\hline $\begin{array}{l}\text { Consulta programada } \\
\text { Idade do cuidador } \\
\text { Situação profissional } \\
\text { Local de prestação de cuidados } \\
\mathrm{N}^{\circ} \text { consultas }\end{array}$ & $\begin{array}{l}p=0,013 \\
p<0,001 \\
p=0,008 \\
p=0,034\end{array}$ & $\begin{array}{l}p=0,034 \\
p=0,920 \\
p=0,828 \\
p<0,001\end{array}$ \\
\hline $\begin{array}{l}\text { Consulta do próprio dia } \\
\text { Idade do cuidador } \\
\text { Causa de morte } \\
\mathrm{N}^{\circ} \text { consultas }\end{array}$ & $\begin{array}{l}p=0,021 \\
p=0,035 \\
p<0,001\end{array}$ & $\begin{array}{l}p=0,018 \\
p=0,188 \\
p<0,001\end{array}$ \\
\hline $\begin{array}{l}\text { Serviço de urgência } \\
\text { Tipo de família } \\
\mathrm{N}^{\circ} \text { fatores de risco para PLP } \\
\mathrm{N}^{\circ} \text { consultas }\end{array}$ & $\begin{array}{l}p=0,030 \\
p=0,011 \\
p=0,001\end{array}$ & $\begin{array}{l}p=0,033 \\
p=0,020 \\
p=0,001\end{array}$ \\
\hline $\begin{array}{l}\text { Nº consultas } \\
\text { Habilitações literárias }\end{array}$ & $p=0,040$ & NA \\
\hline $\begin{array}{l}\text { Problemas de saúde } \\
\text { Tempo de prestação de funções } \\
N^{\circ} \text { consultas }\end{array}$ & $\begin{array}{l}p=0,014 \\
p=0,001\end{array}$ & $\begin{array}{l}p=0,020 \\
p<0,001\end{array}$ \\
\hline $\begin{array}{l}\text { Problemas de saúde Capítulo P-Psicológico } \\
N^{\circ} \text { fatores de risco para PLP }\end{array}$ & $p=0,029$ & NA \\
\hline $\begin{array}{l}\text { Referenciação } \\
\text { Idade do cuidador } \\
\text { Habilitações literárias } \\
\mathrm{N}^{\circ} \text { consultas }\end{array}$ & $\begin{array}{l}p=0,051 \\
p=0,028 \\
p=0,003\end{array}$ & $\begin{array}{l}p=0,174 \\
p=0,027 \\
p=0,001\end{array}$ \\
\hline $\begin{array}{l}\text { Internamento } \\
\text { Estado civil } \\
\text { Cuidador único }\end{array}$ & $\begin{array}{l}p=0,013 \\
p=0,046\end{array}$ & $\begin{array}{l}p=0,303 \\
p=0,031\end{array}$ \\
\hline $\begin{array}{l}\text { Fármacos novos } \\
\text { Habilitações literárias } \\
\text { Tempo de prestação de funções } \\
\text { Local de prestação de funções } \\
\text { Sexo do doente } \\
N^{\circ} \text { consultas }\end{array}$ & $\begin{array}{l}p=0,010 \\
p=0,051 \\
p=0,021 \\
p=0,001 \\
p<0,001\end{array}$ & $\begin{array}{l}p=0,247 \\
p=0,246 \\
p=0,330 \\
p=0,001 \\
p<0,001\end{array}$ \\
\hline $\begin{array}{l}\text { Fármacos psicotrópicos novos } \\
\text { Sexo do doente } \\
\text { Idade do doente }\end{array}$ & $\begin{array}{l}p=0,006 \\
p=0,026\end{array}$ & $\begin{array}{l}p=0,023 \\
p=0,012\end{array}$ \\
\hline $\begin{array}{l}\text { Alteração dosagem de fármacos } \\
\mathrm{N}^{\circ} \text { consultas }\end{array}$ & $p=0,021$ & NA \\
\hline $\begin{array}{l}\text { Alteração dosagem de fármacos psicotrópicos } \\
\text { Parentesco } \\
N^{\circ} \text { fatores de risco para PLP } \\
N^{0} \text { consultas }\end{array}$ & $\begin{array}{l}p=0,067 \\
p=0,007 \\
p=0,023\end{array}$ & $\begin{array}{l}p=0,066 \\
p=0,016 \\
p=0,056\end{array}$ \\
\hline
\end{tabular}

Legenda: $p=p$-value; negrito = relação estatisticamente significativa; NA = não aplicável. te capítulo nos utentes com mais fatores de risco.

Considerando a probabilidade de referenciação do cuidador a especialidades médicas ou outras, esta era tanto maior quanto menor a escolaridade ou quanto maior o número de consultas realizado ( $p=0,027$; $p=0,001$ ).

Encontrou-se maior probabilidade de internamento no ano após a morte do doente quando o cuidador do doente não era único ( $p=0,031$ ).

Em termos de farmacoterapia, após análise conjunta, apenas o sexo masculino do doente falecido e o maior número de consultas se associou a maior probabilidade de prescrição de, pelo menos, um fármaco novo ( $p=0,001 ; p<0,001)$.

Atendendo apenas aos fármacos psicotrópicos, a probabilidade de prescrição de novo era maior nos enlutados que cuidaram de doentes do sexo masculino e mais novos ( $p=0,023$; $p=0,012$ ).

Em termos de alteração de dosagem de fármacos, apenas o maior número de consultas mostrou associação com maior probabilidade de alteração de dose $(p=0,021)$. Analisando os fármacos psicotrópicos de forma isolada, quanto maior o número de fatores de risco para PLP, maior a probabilidade de alteração de dose $(p=0,016)$. 


\section{DISCUSSÃO}

Estão descritos na literatura os vários benefícios do envolvimento dos médicos de família na prestação de cuidados em fim de vida, nomeadamente melhor qualidade dos cuidados prestados, ${ }^{24}$ maior satisfação dos utentes ${ }^{25}$ e maior probabilidade de morte no domicílio. ${ }^{26-28}$ Cerca de $80,5 \%$ dos doentes que faleceram tiveram algum tipo de contacto com o seu médico de família nos doze meses anteriores à sua morte, tendo sido mais frequente este contacto nos utentes inscritos nas UCSP, sem diferença estatisticamente significativa quando comparado com as USF modelo A e as USF modelo B. Realça-se o facto dos grupos comparados serem assimétricos, o que poderá não ter permitido verificar a existência de diferenças com significado estatístico entre as unidades funcionais (UCSP - 35 óbitos, USF modelo B - 509).

Foram incluídos para análise 308 indivíduos, o que corresponde a cuidadores de $11,6 \%$ dos óbitos ocorridos nos dois ACeS. A amostra poderá ser considerada como baixa, podendo ter contribuído para este valor a aplicação dos critérios de exclusão, mais concretamente o facto de cerca de $20 \%$ dos doentes não terem tido contacto com o seu médico de família no último ano de vida, alguns médicos estarem indisponíveis para a colheita de dados ou por não lhes ser possível identificar o cuidador. A taxa de resposta foi maior nas USF modelo B (11 das 13) e menor nas UCSP (três das 15). Este facto poderá estar relacionado com o próprio modelo de organização e filosofia de trabalho, mais concretamente por nas USF modelo B existirem estruturas específicas que atuam como interlocutoras com autores de trabalhos de investigação, maior sensibilização decorrente de incentivos e prática mais frequente de atividades de investigação na própria unidade.

Os cuidadores tinham uma mediana de 66,5 anos de idade, sendo maioritariamente do sexo feminino (79,2\%), viúvos ou casados/união de facto $(78,5 \%)$, com quatro a doze anos de escolaridade $(40,3 \%)$, inativos profissionalmente $(62,0 \%)$, cônjuges $(56,5 \%)$ ou filhos $(33,8 \%)$ do doente, pertencentes a família unitária $(39,0 \%)$ ou nuclear $(19,2 \%)$, não sendo cuidadores únicos $(59,4 \%)$ e residindo conjuntamente com o doente (71,8\%). Os dados obtidos são sobreponíveis aos descritos a nível nacional, em diferentes regiões e diferentes contextos de prestação de cuidados (Norte, ${ }^{29}$ Cen- tro, ${ }^{30}$ Lisboa e Vale do Tejo ${ }^{31}$ e do Algarve ${ }^{32}$ ), e a nível internacional. ${ }^{33-35}$

No que se refere à análise estatística não foi encontrada diferença entre sexos, provavelmente por baixa representatividade do sexo masculino.

A mediana do tempo de prestação de funções como cuidador foi de 30 meses, sendo que $43,3 \%$ cuidou menos de um ano e $24,7 \%$ mais de três anos. Contrariamente aos estudos nacionais, em que os cuidadores na sua maioria afirmavam cuidar há mais de dois anos, ${ }^{29,32,36}$ no presente trabalho o que se verificou foi que a maioria cuidava há menos de dois anos (64,0\%). Esta diferença estará relacionada com diferenças metodológicas entre os estudos citados e o atual, uma vez que, nos primeiros, a quantificação do tempo de prestação de cuidados foi obtida por autorrelato do cuidador, ao invés do que aconteceu neste estudo, no qual esta quantificação foi fornecida por um profissional de saúde (possível viés de memória e registo).

Verificou-se que um menor tempo de prestação de funções estava associado a uma maior probabilidade de codificação de problemas de saúde de novo nos doze meses após a morte do doente $(p=0,020)$. Esta associação poderá ser explicada pelo facto de, numa fase inicial de prestação de cuidados a um doente, surgirem vários fatores (questões clínicas, sociais, familiares e laborais) que podem atuar como agentes de stress no cuidador e levar a problemas de saúde, podendo presumir-se que cuidadores que prestam cuidados durante mais tempo poderão ter, mais frequentemente, problemas de saúde codificados previamente à morte do doente. Por outro lado, o tipo de trajetória da doença poderá ter um contributo, uma vez que a imprevisibilidade da morte pode condicionar uma menor preparação para a mesma e dificultar o processo de luto do cuidador, aumentando a probabilidade de diagnóstico de novos problemas de saúde pós-morte, em situações de menor duração da prestação de cuidados.

A nenhum cuidador foram aplicados instrumentos de avaliação de PLP ou de apoio ao luto, apesar de a mediana de fatores de risco para PLP apresentada pelos participantes ter sido de três, da prevalência de luto prolongado ser de 10 a $20 \%$ nos enlutados, ${ }^{10,13-15} \mathrm{e} \mathrm{de,} \mathrm{em}$ Portugal, se ter identificado uma prevalência de luto complicado de $28,8 \% .^{3}$ 
Relativamente aos doentes falecidos, 57,5\% eram homens, a mediana da idade foi de 81,0 anos e a média de 77,8 anos; em $31,8 \%$ a causa de morte foi doença oncológica e em 30,5\% foi insuficiência de órgão. A nível nacional, em 2017, a idade média ao óbito foi de 78,2 anos, a principal causa de morte foram as doenças do aparelho circulatório $(29,4 \%)$ e os tumores malignos constituíram $25 \%$ das causas de óbito; existiu um discreto predomínio dos homens (50,3\%). ${ }^{37}$ Analisando os dados apresentados destaca-se a inversão das causas de morte. Neste estudo a principal causa de morte foi a doença oncológica. Vários fatores podem justificar esta diferença, nomeadamente o facto de, em 15,3\% dos doentes falecidos, a causa ser desconhecida. Tradicionalmente a doença oncológica apresenta trajetória de declínio mais previsível com limitação funcional mais acentuada $^{38}$ e que se traduz, mais frequentemente, em maiores necessidades, quer clínicas quer sociais, tanto do doente como do cuidador e, por isso, poderão recorrer de forma mais reiterada ao médico de família, facilitando a identificação do cuidador pelo profissional (e aumentando a representatividade deste grupo neste estudo). Esta previsibilidade da trajetória da doença é menos comum nas situações de insuficiência de órgão ou doença neurodegenerativa, dificultando a identificação de cuidador. As doenças cardiovasculares poderão estar subrepresentadas neste estudo por frequentemente serem causa de morte súbita, não existindo cuidador associado.

No que se refere ao acompanhamento clínico dos cuidadores no primeiro ano após a morte do doente, a maioria foi acompanhada só pelo seu médico de família. A baixa taxa de acompanhamento em psiquiatria e psicologia poderá estar subquantificada, uma vez que o cuidador poderá ter recorrido a entidades privadas para obtenção deste tipo de cuidados, não tendo informado o médico de família ou não tendo este registado esse acompanhamento. O recurso aos serviços de saúde privados justifica-se pelo tempo de espera para primeira consulta de psiquiatria no hospital de referenciação - 122 dias em média ${ }^{39}$ - e para a primeira consulta de psicologia nos ACeS. Relativamente a este último aspeto existiam, nos dois ACeS, um psicólogo para cada 50.000 inscritos quando o recomendado é de um para cada 5.000 inscritos. ${ }^{40}$ A mediana do número de consultas com o médico de família foi de quatro. Cerca de
$95,1 \%$ dos cuidadores que tiveram consulta recorreram a consulta programada e $56,8 \%$ a consulta do próprio dia. Destaca-se o facto de a maioria dos internamentos ser por causa cirúrgica eletiva, possivelmente traduzindo a necessidade dos cuidadores adiarem intervenções clínicas, por exemplo procedimentos cirúrgicos. Em $82,5 \%$ dos cuidadores não foi efetuada nenhuma referenciação e em 15,3\% ocorreu referenciação para uma especialidade médica. Sobressai a elevada taxa de referenciação neste primeiro ano após a morte do doente quando comparada com a literatura nacional - 10,1\% ${ }^{41}$ -, o que poderá refletir não só a indisponibilidade do cuidador para cuidar dos seus próprios problemas de saúde, como acima referido, mas também o desenvolvimento de novos problemas de saúde. Quanto a este último aspeto foram identificados 335 novos problemas de saúde em $58,1 \%$ dos cuidadores, sendo que $11,0 \%$ dos problemas eram do foro psicológico.

Estatisticamente verificou-se que o sexo masculino do doente falecido se associou a maior probabilidade de prescrição de, pelo menos, um fármaco novo ao cuidador $(p<0,001)$; atendendo apenas aos fármacos psicotrópicos, a probabilidade de prescrição de novo era maior nos enlutados que cuidaram de doentes do sexo masculino e mais novos ( $p=0,023 ; p=0,012$ ). Apenas o maior número de consultas mostrou associação com maior probabilidade de alteração de dose de qualquer classe de fármaco ( $p=0,021)$. De acordo com estes resultados, aparentemente terá um impacto diferente e no sentido negativo a circunstância de o doente ser homem e mais novo. O papel social do homem nas famílias portuguesas e o facto de ainda representar a principal fonte de rendimentos poderá justificar esta associação. A idade jovem do doente falecido constitui um fator de risco para PLP, como consta da checklist apresentada na Norma. ${ }^{17}$ Outros fatores de risco descritos na Norma e prevalentes nesta amostra, como cuidador do sexo feminino e cônjuge do falecido, não revelaram associação estatisticamente significativa com as variáveis em estudo.

No que se refere à temática central do estudo - a abordagem do luto ao nível dos cuidados de saúde primários -, pode inferir-se que são desconhecidas ou inexistentes as intervenções realizadas pelos médicos de família no processo de luto (aplicação de instrumentos de avaliação de risco no luto e/ou fornecimento de 
ferramentas de apoio ao luto) e, aparentemente, não existiu uma abordagem por níveis aos enlutados. O luto, no seu primeiro ano, foi «medicalizado» (em 35,1\% dos cuidadores ocorreu introdução e/ou alteração de dose de fármacos psicotrópicos), não tendo ocorrido referenciação, na mesma proporção, para psicologia e/ou psiquiatria. Estes dados reforçam os apresentados no relatório do Conselho Nacional de Saúde (Sem mais tempo a perder: saúde mental em Portugal - Um desafio para a próxima década). Portugal regista a $5^{\text {a }}$ posição na OCDE no consumo de medicamentos antidepressivos e um consumo "preocupante» de ansiolíti$\cos .^{42} \mathrm{~A}$ introdução de fármacos nesta fase de vida, particularmente vulnerável, sem recurso a outras medidas de apoio/terapêuticas, poderá contribuir para este problema nacional. Esta abordagem poderá refletir, por um lado, a desadequação do número de profissionais de psicologia nos cuidados de saúde primários e, por outro, a formação insuficiente dos médicos de família para a abordagem e orientação do processo de luto, o tempo de consulta desadequado ao nível dos cuidados de saúde primários para a prestação de cuidados, o número excessivo de utentes inscritos por médico de família, tornando-se, assim, mais rápido medicar do que efetuar uma intervenção global e multidisciplinar.

Como pontos fortes deste trabalho destacam-se o tema inovador e pertinente, a inexistência de estudos semelhantes em Portugal, o tamanho amostral obtido e o processo de colheita e análise de dados, uma vez que este foi realizado por duas das autoras, de forma independente, preservando a consistência interna dos resultados.

Por outro lado, identificam-se, como limitações, a baixa taxa de resposta e o possível viés de seleção, de memória e de registo na obtenção dos dados. Existiram grupos entre os quais não foi possível estabelecer comparações por tamanho amostral assimétrico, podendo, assim, existir associações estatisticamente significativas que não foram demonstradas.

\section{CONCLUSÃO}

Este trabalho possibilitou caracterizar os cuidadores, do ponto de vista sociodemográfico e clínico, nos doze meses que se sucederam à morte do doente.

Esta caracterização permite refletir sobre os diferentes aspetos da vulnerabilidade do cuidador, da pro- cura de cuidados em determinada fase/etapa da sua vida ou/e em situação de crise familiar e da resposta que lhe é proporcionada pelo seu médico de família. Atendendo ao papel essencial deste no apoio no luto poderá ser útil o desenvolvimento de ações formativas, a elaboração de manuais de boas práticas sobre apoio no luto ao nível dos cuidados de saúde primários, bem como a definição de indicadores de processo e resultado na área do luto.

Permanecem desconhecidos muitos aspetos, que deverão ser alvo de outros estudos, como: perceber se os médicos de família portugueses identificam os doentes em fim de vida, se, nessa circunstância, adequam os seus cuidados ao doente e ao cuidador e que tipo de apoios/recursos de saúde e comunitários são disponibilizados ao cuidador, previamente e após a morte do doente.

\section{REFERÊNCIAS BIBLIOGRÁFICAS}

1. Barbosa A, Pina PR, Tavares F, Neto IG. Manual de cuidados paliativos. $3^{\text {a }}$ ed. Lisboa: Faculdade de Medicina da Universidade de Lisboa; 2016. ISBN 9789729349379

2. Nunes L. E quando eu não puder decidir? Lisboa: Fundação Francisco Manuel dos Santos; 2016. ISBN 9789898838698

3. Coelho A, Delalibera M, Barbosa A, Lawlor P. Prolonged grief in palliative family caregivers: a pilot study in a Portuguese sample. Omega (Westport). 2015;72(2):151-64.

4. Krug K, Miksch A, Peters-Klimm F, Engeser P, Szecsenyi J. Correlation between patient quality of life in palliative care and burden of their family caregivers: a prospective observational cohort study. BMC Palliat Care. 2016;15:4.

5. Cohen LM, Germain MJ. Caregiver burden and hemodialysis. Clin J Am Soc Nephrol. 2014;9(5):840-2.

6. Santos PR, Santos IM, Freitas Filho JL, Macha CW, Tavares PG, Portela $A C$, et al. Emotion-oriented coping increases the risk of depression among caregivers of end-stage renal disease patients undergoing hemodialysis. Int Urol Nephrol. 2017;49:1667-72.

7. Delalibera M, Presa J, Barbosa A, Leal I. Sobrecarga no cuidar e suas repercussões nos cuidadores de pacientes em fim de vida: revisão sistemática da literatura [Burden of caregiving and its repercussions on caregivers of end-of-life patients: a systematic review of the literature]. Ciênc Saúde Colet. 2015;20(9):2731-47. Portuguese

8. Lei n. ${ }^{\circ}$ 100/2019, de 6 de setembro. Diário da República. I Série;(171).

9. Götze H, Brähler E, Gansera L, Schnabel A, Gottschalk-Fleischer A, Köhler N. Anxiety, depression and quality of life in family caregivers of palliative cancer patients during home care and after the patient's death. Eur J Cancer Care. 2018;27(2):e12606.

10. Hudson P, Hall C, Boughey A, Roulston A. Bereavement support standards and bereavement care pathway for quality palliative care. Palliat Support Care. 2018;16(4):375-87.

11. Näppä $U$, Lundgren $A B, A x e l s s o n ~ B$. The effect of bereavement groups 
on grief, anxiety, and depression: a controlled, prospective intervention study. BMC Palliat Care. 2016;15:58.

12. Waller A, Turon H, Mansfield E, Clark K, Hobden B, Sanson-Fisher R. Assisting the bereaved: a systematic review of the evidence for grief counselling. Palliat Med. 2016;30(2):132-48.

13. Breen LJ, Aoun SM, O'Connor M, Rumbold B. Bridging the gaps in palliative care bereavement support: an international perspective. Death Stud. 2014;38(1-5):54-61.

14. Morris SE, Block SD. Adding value to palliative care services: the development of an institutional bereavement program. J Palliat Med. 2015;18(11):915-22.

15. Sealey M, O'Connor M, Aoun SM, Breen LJ. Exploring barriers to assessment of bereavement risk in palliative care: perspectives of key stakeholders. BMC Palliat Care. 2015;14:49.

16. American Psychiatric Association. Diagnostic and statistical manual of mental disorders (DSM - 5). 5th ed. Washington:APA Publishing; 2013. ISBN 9780890425558

17. Direção-Geral da Saúde. Modelo de intervenção diferenciada no luto prolongado em adultos: norma n. ${ }^{\circ}$ 003/2019, de 23/04/2019. Lisboa: DGS; 2019.

18. Gómez-Batiste X, Connor S. Building integrated palliative care programs and services. Barcelona: La Caixa Banking Foundation; 2017. ISBN 9788497666022

19. Murray SA, Firth A, Schneider N, Van den Eynden B, Gomez-Batiste X, Brogaard $T$, et al. Promoting palliative care in the community: production of the primary palliative care toolkit by the European Association of Palliative Care Taskforce in primary palliative care. Palliat Med. 2015;29(2):101-11.

20. Instituto Nacional de Estatistica. Censos 2001: metodologia [homepage]. Lisboa: INE; 2014 [cited 2019Aug 15]. Available from: https://censos.ine.pt/xportal/xmain?xpid=CENSOS\&xpgid=censos_metodologia

21. WONCA. Classificação internacional de cuidados de saúde primários - ICPC-2E v4.4 PT. 2 [Internet]. Lisboa: Administração Central do Sistema de Saúde; 2016. Available from: http://www.acss.min-saude.pt/wpcontent/uploads/2016/07/1_ICPC_2_4_4_VF.pdf

22. General Assembly of the World Medical Association. World Medical Association Declaration of Helsinki: ethical principles for medical research involving human subjects. J Am Coll Dent. 2014;81(3):14-8.

23. Neto M, Pimentel JP, Durval M, Araújo FO, Guerreiro AC. Perfil local de saúde 2017: ACeS Gaia e Espinho/Gaia [Internet]. Porto: ARS Norte; 2017.Available from:https://bicsp.min-saude.pt/pt/biufs/1/10023/Documents/PeLS2017_A17_GaiaEspinho.pdf

24. Aabom B, Kragstrup J, Vondeling H, Bakketeig LS, Stovring H. Does persistent involvement by the GP improve palliative care at home for endstage cancer patients? Palliat Med. 2006;20(5):507-12.

25. Mitchell GK. How well do general practitioners deliver palliative care? A systematic review. Palliat Med. 2002;16(6):457-64.

26. Fukui S, Fukui N, Kawagoe H. Predictors of place of death for Japanese patients with advanced stage malignant disease in home care settings: a nationwide survey. Cancer. 2004;101(2):421-9.

27. Gomes B, Higginson IJ. Factors influencing death at home in terminally ill patients with cancer: systematic review. BMJ. 2006;332(7540):51521.

28. Neergaard MA, Vedsted P, Olesen F, Sokolowski I, Jensen AB, Sønder- gaard J. Associations between home death and GP involvement in palliative cancer care. Br J Gen Pract. 2009;59(566):671-7.

29. Pires C, Teixeira L, Ribeiro O, Oliveira S. Custo dos cuidados informais a pessoas idosas da região Norte de Portugal: aplicação do método do bem substituto. Actas Gerontol. 2016;2(1):1-7.

30. Pocinho R, Belo P, Melo C, Navarro-Pardo E, Fernández-Muñoz JJ. Relação entre o estado psicossocial do cuidador informal e o tempo de cuidado dos idosos da região centro de Portugal [Relation between informal caregivers and the time spent on elder care in the centre of Portugal]. Educ Humanismo. 2017;19(32):88-101. Portuguese

31. Broeiro-Gonçalves P. Características dos cuidadores de idosos assistidos pelas equipas domiciliárias da Rede Nacional de Cuidados Continuados Integrados na região de Lisboa e Vale do Tejo: estudo transversal observacional [Caregiver's characteristics of disability elderly cared by home-teams of the National Network of Integrated Care in the Lisbon and Tagus Valley: cross-sectional study]. Saúde Tecnol. 2017;(17):39-46. Portuguese

32. Duarte A, Joaquim N, Lapa F, Nunes C. Qualidade de vida e sobrecarga dos cuidadores informais dos pacientes idosos das unidades de cuidados de assistência domiciliar do Algarve (PT) [Quality of life and burden of informal caregivers of elderly patients of home care assistence in the Algarve (PT)]. SaBios Rev Saúde Biol. 2018;12(1):12-26. Portuguese

33. Adelman RD, Tmanova LL, Delgado D, Dion S, Lachs MS. Caregiver burden: a clinical review. JAMA. 2014;311(10):1052-60.

34. DiGiacomo M, Hatano Y, Phillips J, Lewis J, Abernethy AP, Currow DC. Caregiver characteristics and bereavement needs: findings from a population study. Palliat Med. 2017;31(5):465-74.

35. Konerding U, Bowen T, Forte P, Karampli E, Malmström T, Pavi E, et al. Do caregiver characteristics affect caregiver burden differently in different countries? Am J Alzheimers Dis Other Demen. 2019;34(3):14852.

36. Afonso RM, Tomaz T, Brandão D, Ribeiro O. Cuidadores de idosos centenários na região da Beira Interior (Portugal) [Profiling centenarians' informal carers: An exploratory study in the Beira Interior region (Portugal)]. Anal Psicol. 2019;37(2):147-60. Portuguese

37. Instituto Nacional de Estatística. Causas de morte 2017. Lisboa: INE; 2019.

38. Murray SA, Kendall M, Boyd K, Sheikh A. Illness trajectories and palliative care. BMJ. 2005;330(7498):1007-11.

39. Ministério da Saúde. Tempos médios de resposta para primeiras consultas hospitalares com origem nos cuidados de saúde primários: consulta a tempo e horas [Internet]. Jornal Económico; 2018 [cited 2019 Aug 11]. Available from: www.jornaleconomico.sapo.pt/wpcontent/uploads/2018/01/SNS-2018-Tempos-de-Espera.pdf

40. Ordem dos Psicólogos. Parecer sobre o rácio dos psicólogos [Internet]. Lisboa: Ordem dos Psicólogos; 2017. Available from: http://recursos.ordemdospsicologos.pt/files/artigos/parecer_sobre_o_r_cio_de_psic_lo gos.pdf

41. Ponte CM, Moura BG, Cerejo AC, Braga R, Marques I, Teixeira A, et al. Referenciação aos cuidados de saúde secundários [Referral to secondary care]. Rev Port Med Geral Fam. 2006;22(5):555-68. Portuguese

42. Conselho Nacional de Saúde. Sem mais tempo a perder - Saúde mental em Portugal: um desafio para a próxima década [Internet]. Lisboa: 
CNS; 2019. Available from: http://www.cns.min-saude.pt/wp-content/uploads/2019/12/SEM-MAIS-TEMPO-A-PERDER.pdf

\section{CONFLITO DE INTERESSES}

Os autores declaram não ter quaisquer conflitos de interesse.

\section{FINANCIAMENTO DO TRABALHO}

Os autores declaram não ter recebido quaisquer financiamentos.
ENDEREÇO PARA CORRESPONDÊNCIA

Soraia Santos

E-mail: soraia.reis.santos@gmail.com

https://orcid.org/0000-0002-5398-3560

Recebido em 04-01-2020

Aceite para publicação em 25-09-2020

\section{ABSTRACT}

\section{BEREAVEMENT IN CAREGIVERS: THE REALITY OF TWO HEALTH CENTER UNITS OF NORTH OF PORTUGAL}

Aim: Characterize caregivers in the 12 months after the patient's death (sociodemographic data, clinical follow-up, health problems, and pharmacological therapy).

Methods: We conducted an observational, retrospective, and analytical study at the primary health care level. The population consisted of caregivers, identified by the family doctor, of the deceased patients in 2017. After sending an invitation to the participation of all Functional Units, data were obtained that allowed characterizing the caregivers and clinical follow-up in the first year of bereavement through the family physicians who agreed to participate. For the descriptive analysis, we used absolute and relative frequencies, median, minimum, and maximum.

Results: Of the 308 caregivers, we highlight the representativeness of the elderly (median age - 66.5 years) and women (79.2\%), as well as the exclusive follow-up by the family doctor in $93.2 \%$ of the situations. New prescribing and/or dosage changes of psychotropic drugs occurred in $35.1 \%$ of caregivers, contrasting with the low follow-up rate in psychology (3.5\%) and/or psychiatry (4.2\%) and the lack of evaluation of risk in bereavement and provided support tools.

Conclusion: The characteristics of the caregivers agreed with those described in the literature. The medicalization of mourning may reflect a lack of training, consultation time, and resources of psychology and psychiatry. The essential role of the family doctor in supporting bereavement is proved; the implementation of institutional and organizational measures can improve bereavement care.

Keywords: Primary health care; Bereavement; Caregivers; Family physicians. 